\title{
The Internet As a Medium for Online Instruction
}

\section{Rama Vishwanatham, Walter Wilkins, and Thomas Jevec}

\begin{abstract}
A task force of librarians developed an introductory course on the Internet which was conducted over the university's e-mail network. This sixteen-lesson course was distributed via listserv to students, staff, residents, and faculty, some using university facilities and some using equipment at their home or office, in various locations throughout the state. Initially, more than 450 people subscribed. Subscribers received two lessons a week for eight weeks. Lessons were made available via FTP or gopher after initial distribution. Content included basic information, troubleshooting advice, and exercises for practice. Pre- and post-tests were conducted via listserv to assess the participants' prior knowledge and subsequent knowledge gain. This article describes the development of the online course. It also discusses the results of pre- and posttests, and the implications for the library's instructional role of using an online instruction medium.
\end{abstract}

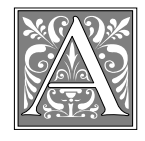

$s$ more information resources become available onlinewhether on local networks, Intranets, or the Internet-librarians have turned their attention to the training required to navigate networks successfully. Yet, as the Internet changes and evolves, networked information instruction needs and resources are in a constant state of flux. Instructional demands on librarians are changing as the focus on instruction shifts from print to electronic resources. As libraries provide more networked access to resources, more use of library services occurs outside the library; there are more "remote users." Remote users often need special assistance in finding and using the electronic information resources. This paper focuses on how the Internet can itself be used as a medium of instruction uniquely suited to reach remote users.

Computer-assisted instructional programs for bibliographic instruction have been in use in libraries for a number of years. Typically, users must come to the library or some other central location to receive instruction. Remote users are not easily reached by in-per-

Rama Vishwanatham is an Assistant Reference Librarian and Assistant Professor in the Library of the Health Sciences (Chicago) at the University of Illinois at Chicago; e-mail: rama@uic.edu. Walter Wilkins is an Assistant Health Sciences Librarian and Assistant Professor at the Library of the Health Sciences (Peoria) at the University of Illinois at Chicago; e-mail: walter@uic.edu. Thomas Jevec is an Electronic Services Librarian and Assistant Professor in the University Library at the University of Illinois at Chicago; e-mail: tej@uic.edu. 
son instruction sessions. Julie Sih and Christy Hightower found that a significant number of their clientele failed to participate in instruction sessions, despite expressed need or interest. ${ }^{1}$ The chief barrier to participation was the difficulty in finding time to come to the library for instruction. There also appeared to be an issue of privacy: some expressed reluctance to attend group sessions where their ignorance or in-

\section{Once such technical needs are no longer paramount, users become more concerned with broader issues of the relevance of Internet re- sources to their work or lifestyle.}

ability might be exposed to colleagues and staff.

This apparent reluctance to attend group sessions has been noted by Karen Wielhorski, who suggests that the computer-based setting of information retrieval tends to split users into two groups based on their perceived expertise. ${ }^{2}$ One group might be called "techies," or experts. Already familiar with the systems, techies tend to be impatient with formal instruction and prefer detailed descriptions or instruction sheets they can figure out for themselves. The other group might be considered "technologically challenged," as they consider themselves to be unfamiliar with either the system in use or computers in general. This group desires specific examples, detailed instruction, and the opportunity to try things out one step at a time with supportive coaching or counseling. Sih and Hightower, as well as Wielhorski, suggest that differences in skill levels and information needs are bound to limit the effectiveness of group instruction. ${ }^{3}$ They concluded that the only alternative is unscheduled point-of-use instruction.

In addition to meeting the informa- tion needs of the various users, the instruction must be designed to accommodate the structure of the subject matter. According to John S. Makulowich, there is no shortage of resources for developing instruction on the use of the Internet. ${ }^{4}$

It may not be either easy or desirable to develop a comprehensive design or content for instruction. Many who have developed instructional programs find that content frequently must be revised. Lisa A. Oberg refers to Internet instruction as a "moving target," in that the subject matter seems to be constantly changing. ${ }^{5}$ Wielhorski suggests that users need both procedural knowledge, techniques for utilizing Internet features, and conceptual knowledge, principles for making use of Internet resources. ${ }^{6}$ Users initially may express needs for procedural knowledge, or techniques, focusing on the Internet or the computer. Once such technical needs are no longer paramount, users become more concerned with broader issues of the relevance of Internet resources to their work or lifestyle. Thus, the user as well as the Internet are both "moving targets."

In planning to design instruction for "moving targets," Edmund F. SantaVicca's proposal for a modular outline of the Internet makes the point that the Internet is primarily an information resource and that instructional goals might be best conceived as helping meet needs for access to information:

I. Internet Functions

II. Typology of Traditional Information Services

III. Typology of Internet Information Sources

IV. Evaluating Traditional Information Sources

V. Evaluating Internet Information Queries

VI. Typology of Information Queries VII. Additional Issues ${ }^{7}$

Many current instructional programs ini- 
tially focus on categories I through III, and may be considered procedural knowledge. Broader issues of what Wielhorski calls conceptual knowledge may be included in SantaVicca's categories IV through VI. ${ }^{8}$

According to Susan J. Barnes, users perceive their needs primarily for hands-on instruction, or Wielhorski's procedural knowledge. ${ }^{9}$ Yet Dianne Rothenberg reports that the ability to search the Internet is becoming recognized as a useful skill. ${ }^{10}$ Searching may be popularly thought of as only a technique, but little progress may be made in searching without some familiarity with the typology and relative value of information sources, as in SantaVicca's outline. ${ }^{11}$

Sih and Hightower report on a program developed in 1994, at the University of California-San Diego, to teach use of the INSPEC database, using six lessons distributed weekly by e-mail. ${ }^{12}$ Though many current instructional programs focus exclusively on "Internet Functions" and follow a group seminar format, there are few reported examples of instructional programs using electronic communication to reach remote users. ${ }^{13}$

\section{Methodology}

The possibility of providing instruction to library users, incorporating both procedural and conceptual knowledge, without requiring them to come to the library is the focus of this paper. Specifically, this paper discusses the results and implications of using an online instruction medium to fulfill the library's instructional role based on the University of Illinois at Chicago (UIC) experience.

UIC consists of a main library and four health sciences libraries (located in Chicago, Rockford, Peoria, and Urbana) and an art, science, and mathematics library. It is primarily an urban campus with almost 25,000 undergradu- ate and graduate students, most of whom are commuters. The UIC libraries had offered sessions on using the Internet at traditional on-site instructional sessions and attendance was lower than hoped for. Few people signed up and still fewer came to scheduled sessions. The average session, held weekly, was attended by only about 10 people. Separate sessions offered at the various campuses were both timeconsuming and costly. Realizing that meeting the varied needs of users was not cost-effective with this traditional model, in November 1993 the UIC libraries decided to form the Internet Training Class Task Force. This body was charged with developing and offering an online Internet course to be administered over the university's computer network via the Internet.

The task force first considered the issues of the remote users, information needs of the various levels of users, and the design of the course itself. The online model of Internet instruction seemed particularly appropriate to UIC where a large percentage of students, staff, and faculty already had access to the campus e-mail network and Internet from their homes and dorms. The remote model allowed participants to attend the course easily at their desktops, without having to go to the library or elsewhere for a class. There was none of the "inconvenience of scheduled training classes."14 It was expected that the flexibility of online participation might lead to increased participant attendance and satisfaction.

The task force developed a course designed to provide instruction on use of the Internet in the UIC computer network environment. The course was designed to be "procedural." 15 It was called "Ride the eTrain: An introduction to the Internet at UIC" (eTrain). The course was scheduled to be conducted during the fall semester of 1994. At this time, the World Wide Web was just be- 


\begin{tabular}{|c|}
\hline $\begin{array}{l}\text { FIGURE } 1 \\
\text { PreCourse Survey }\end{array}$ \\
\hline Ride The eTrain: An Introduction To The Internet At UIC \\
\hline $\begin{array}{l}\text { 1. How often have you used the Internet in the past six months? (mark an X) } \\
\text { never ___ monthly ___ daily }\end{array}$ \\
\hline $\begin{array}{l}\text { 2. How would you rate your Internet expertise?(circle or mark an } \mathrm{X} \text { to indicate your } \\
\text { choice) }\end{array}$ \\
\hline 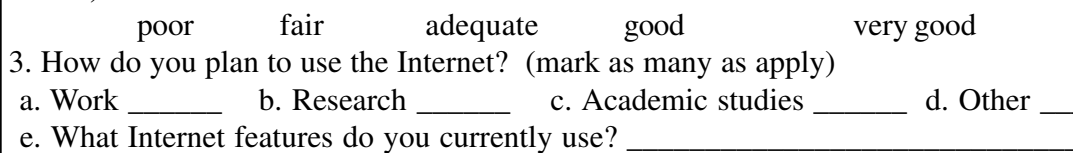 \\
\hline
\end{tabular}

4. Where do you do most of your Internet or e-mail work?
a. Home
b. Computer lab
c. Office
d. Other

5. What is your status at $\overline{\text { UIC? }}$
a. Undergraduate student
b. Graduate student
d. Faculty
e. Alumni

c. Staff

f. Other

6. Where did you hear about Ride the eTrain?

coming widely available and was not yet ubiquitous on the UIC campus. For this reason, the course focused on using the Internet from a remote host environment with access through an IBM mainframe computer.

The target audience was as broad as possible, and the intent was to make this program available to all UIC students, faculty, and staff who had Internet access through the university. The program was to be accessible on all campuses in four different cities. The course was distributed by a listserv to reach the maximum number of users.

In addition to trying to reach the maximum number of users, this form of online instruction had other advantages. The distribution of lessons to the e-mail accounts of course participants permitted instruction to take place wherever and whenever participants used the Internet. Thus, participants did not have to come to a central location or appear at a particular time in order to participate. Subscription to the course was restricted to UIC faculty, staff, and students, and the list was closed and monitored.

Distributed lessons could be reviewed as often as participants wished, printed out, downloaded, or ignored if not relevant to their particular needs. Access to instruction could be available at the convenience of the participant, but at the expense of direct feedback or interaction with the instructor. Opportunities for feedback were provided via a discussion group, eTrain-1, open to all participants who were able to communicate and help each other. Course instructors also monitored the listserv to provide assistance when needed. In addition, detailed instructions for contacting instructional staff or requesting specific help were provided at the end of each lesson.

\section{Design Issues in Online Instruction}

In designing the course these four objectives were included:

1. Local platform emphasis: To pro- 


\section{FIGURE 2 \\ PostCourse Survey}

Ride The eTrain: An Introduction To The Internet At UIC

Ride The eTrain Evaluation

A. Course Information (circle or $\mathrm{X}$ out the number of your response)

1. Level of subject treatment

\begin{tabular}{|c|c|c|c|c|}
\hline poor & fair & adequate & good & very good \\
\hline 1 & 2 & 3 & 4 & 5 \\
\hline
\end{tabular}

2. Organization of the course

3. Content of the course

$\begin{array}{lll}1 & 2 & 3\end{array}$

4. Examples and exercises

12

12

$3-4$

5. Relevant to your own needs

12

3

$4 \quad 5$

6. Length of the course

12

3

$4 \quad 5$

$\begin{array}{lll}3 & 4 & 5\end{array}$

7. If your answer to question 6 is 1 or 2 , should the course be (mark an $\mathrm{X}$ in the line)

8. How would you compare Ride the eTrain

to a traditional workshop or seminar? $\quad 1 \quad 2$

9. How convenient was this course, compared to attending
a traditional workshop or seminar?
12
3
4
5

B. Participant Information

1. How often did you use the Internet in the six months prior to participating in Ride the eTrain? (mark an X) never monthly weekly daily

2. How would you rate your Internet expertise AFTER
participating in Ride the eTrain?
12
34
5

3. There were 7 primary sections in this course. Please rate their value to your own use of the Internet: (circle or $\mathrm{X}$ out the number of your response)

$\begin{array}{llllll}\text { 3a. The Internet (1-4) } & 1 & 2 & 3 & 4 & 5 \\ \text { 3b. Electronic mail (5) } & 1 & 2 & 3 & 4 & 5 \\ \text { 3c. Discussion groups (6) } & 1 & 2 & 3 & 4 & 5 \\ \text { 3d. TELNET (7-8) } & 1 & 2 & 3 & 4 & 5 \\ \text { 3e. FTP (9-10) } & 1 & 2 & 3 & 4 & 5 \\ \text { 3f. Gopher (11-13) } & 1 & 2 & 3 & 4 & 5 \\ \text { 3h. Exploring further (14) } & 1 & 2 & 3 & 4 & 5\end{array}$

4. How do you plan to use the Internet? (mark as many as apply)
a. Work
b. Research
c. Academic studies
d. Personal use
e. Other

5. Where do you do most of your Internet or e-mail work?
a. Home
b. Computer lab
c. Office
d. Other

6. What is your status at UIC?
a. Undergraduate student
b. Graduate student
c. Staff
d. Faculty
e. Alumni
f. Other

7. How long have you been at UIC?

8. Where did you hear about Ride the eTrain?

9. Please feel free to add any comments or suggestions below or in a separate message sent tou11556@uicvm. 


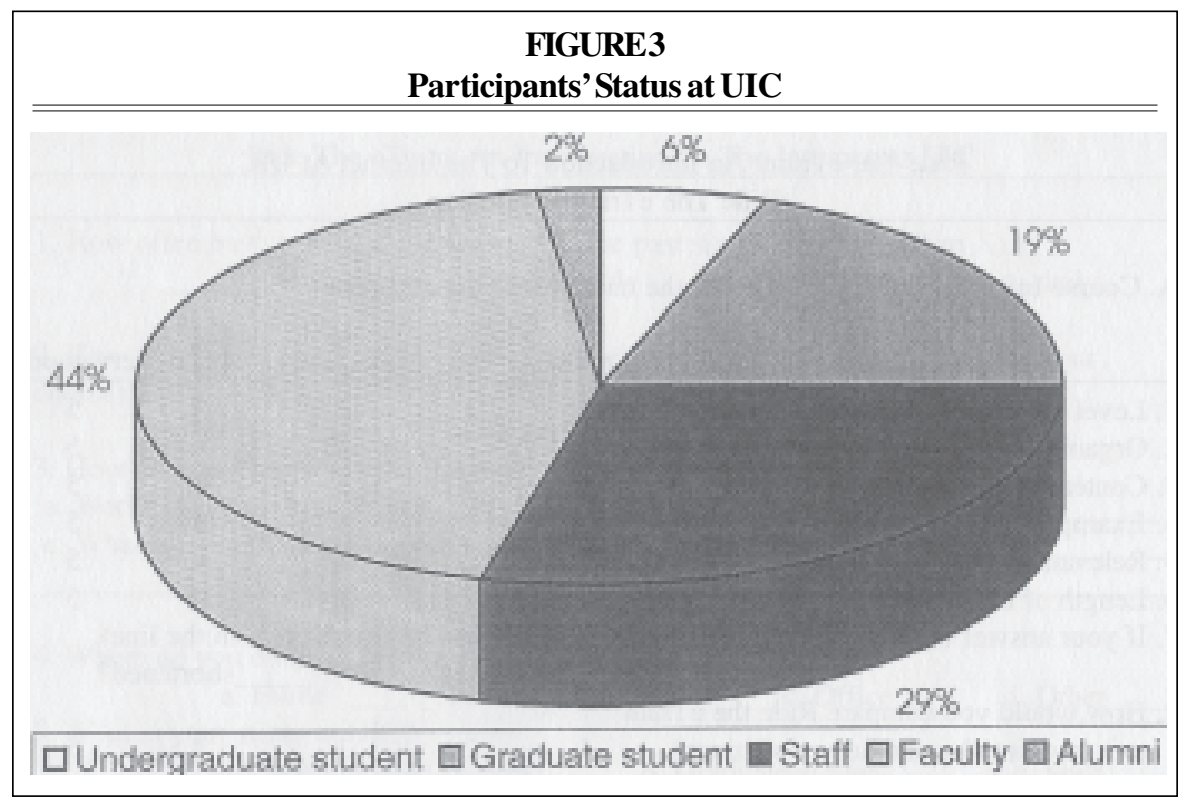

vide instruction to UIC faculty, staff, and students who were using the Internet from the UIC mainframe. Thus, detailed consideration of the other forms of Internet access was deliberately avoided.

2. Consistency in lesson format: To construct each lesson using a similar format. The course and the lessons proceeded from general discussion to specific details. Every lesson followed a similar structure:

- List of objectives

- General definitions and descriptions

- Specific examples

- References to additional information

- Troubleshooting advice

3. Conciseness in length: To avoid having the participants page through several screens. The use of examples and instructions in procedures was kept to a minimum so as to encourage viewing the entire lesson. The purpose was not only to keep the lesson brief, but also to avoid the impression that Internet communication was overly complicated or difficult to master.
4. Encouragement of exploration: To encourage participants to explore further on their own. Participants were consistently referred to additional sources of help or online documentation, both in the text of the lessons and at a separate section at the lesson's conclusion. The footer of each lesson also encouraged participants to contact the course developers via e-mail or to contact the help desk at the University Computer Center. Encouragement to explore and references to collateral material were always accompanied by the invitation to contact a specified individual for assistance.

The task force reviewed and evaluated a number of online Internet instruction classes available at that particular time. None of them was detailed or specific enough to help the users in the UIC environment.

\section{Course Content Organization}

The task force determined that this program should offer procedural information about the Internet, instruction in basic skills in using the Internet, and 


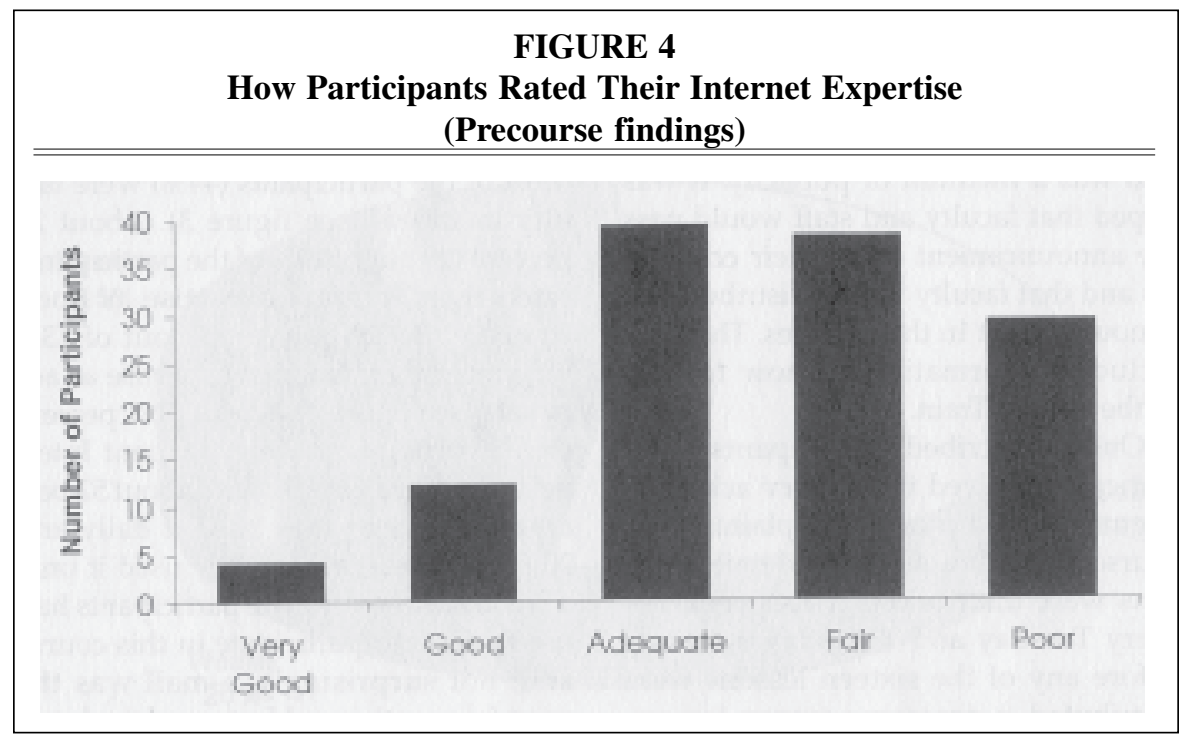

general advice on troubleshooting. The underlying principle was that the course materials should serve as a starting point from which users would gain the skills to teach themselves and remain up to date with the applications relevant to their needs.

The information offered was essentially descriptive in nature and began with a brief history of the Internet. Major types of Internet access were described in detail. Instead of taking a "frequently asked questions" (FAQ) approach, the lessons were written using brief, detailed narratives. The point of the initial lessons was to develop a general understanding of the Internet as an information access system.

Internet skills were the focus of the remainder of the lessons. These lessons focused on mastering specific procedures necessary to initiate communication services. Each lesson began with a statement of the lesson objective. A brief explanation was then followed by an illustration of the use of the application. Following the illustration, an example or exercise was presented. For long procedures, a list of steps to follow was added for review and reinforce- ment. Troubleshooting tips rounded out each lesson. These tips were confined to common or typical problems faced by novice users, and advice was offered as problem-solving examples. The skills instruction concentrated on specific command syntax whereas the troubleshooting advice demonstrated that Internet problems usually have solutions.

The course included the following lessons:

1. Welcome to "Ride the eTrain"

2. What Is the Internet?

3. The Evolution of the Internet

4. How \& Why the Internet Works

5. Electronic Mail

6. Discussion Groups

7. Introduction to Remote Login: Telnet

8. Using Telnet

9. Introduction to File Transfer: FTP

10. Using FTP

11. Introduction to GOPHER at UICVM

12. Basic Internet Tour with GOPHER

13. Advanced Internet Tour with GOPHER

14. Exploring the Internet Further: Im ages, Sound, Graphics, and Much More!

15. Evaluation 
16. Where the eTrain Has Been

The basic information content of this course depicts features of the Internet as part of a larger communication system. Each lesson attempted to balance the three types of content, as appropriate for the subject at hand.

\section{Making the Course Accessible}

The course was publicized throughout the campuses. Posters and flyers were posted in various locations, including student unions, libraries, and college departments. The campus newspapers, UIC News and Chicago Flame, ran editorial announcements. The faculty and staff discussion listserv on campus, Academy, also was a medium of publicity. It was hoped that faculty and staff would pass the announcement on to their coworkers and that faculty would distribute the announcement in their classes. The flyer included information on how to subscribe to the eTrain.

Once subscribed, participants automatically received the listserv acknowledgment and a message explaining the course and administrative details. Lessons were distributed twice weeklyevery Tuesday and Thursday morning. Before any of the sixteen lessons were distributed, a precourse survey (an optional participant survey) was distributed to collect information on the level of Internet exposure and use by the participants (see figure 1). This helped course administrators gauge the participants' Internet skill level as well as obtain a self-assessment of their Internet expertise. At lesson fifteen, a postcourse survey was mailed to participants to evaluate the course's effectiveness and their level of improvement (see figure 2).

After distribution to the listserv, lessons were archived on the campus gopher and FTP sites (and later on the Web site) for access by nonsubscribers and participants wishing to review lessons without having to save them in their e-mail space. Access to the eTrain through gopher was available only to non-UIC users in this manner. In addition, eTrain lessons were available to UIC users through the university's USENET service, NetNews. Moreover, a practice FTP site was established for users to perform exercises in a "safe" environment.

\section{Results}

\section{Pre- and Postcourse Surveys}

The first "Ride the eTrain" lesson was available in September 1994. More than 450 people subscribed. Before the course started, the optional precourse survey was administered; 135 participants responded for a return rate of 30 percent. Most of the participants (44\%) were faculty members (see figure 3 ). About 21 percent (29 out of 135) of the participants rated their Internet expertise as poor, compared to 29 percent (39 out of 135) who rated their Internet expertise as adequate (see figure 4). About $72 \%$ percent of the participants were frequent Internet users (see figure 5), with about 52 percent responding they used it daily and 20 percent responding they used it only a few times a week. The participants had used email to participate in this course and, not surprisingly, e-mail was the most frequently used feature of the Internet, with about 50 percent (67 out of 135) of the participants saying they used it regularly (see figure 6).

The eTrain lessons were distributed over a period of eight weeks. At the end of that period, the postcourse survey showed the positive results of the course. The postcourse survey had an 8 percent return rate (35 out of 450 participants). After completion of the course, the rate of Internet expertise had risen from adequate to good (see figure 7).

The precourse survey revealed that 60 percent ( 81 out of 135 ) of the participants intended to use the Internet pri- 


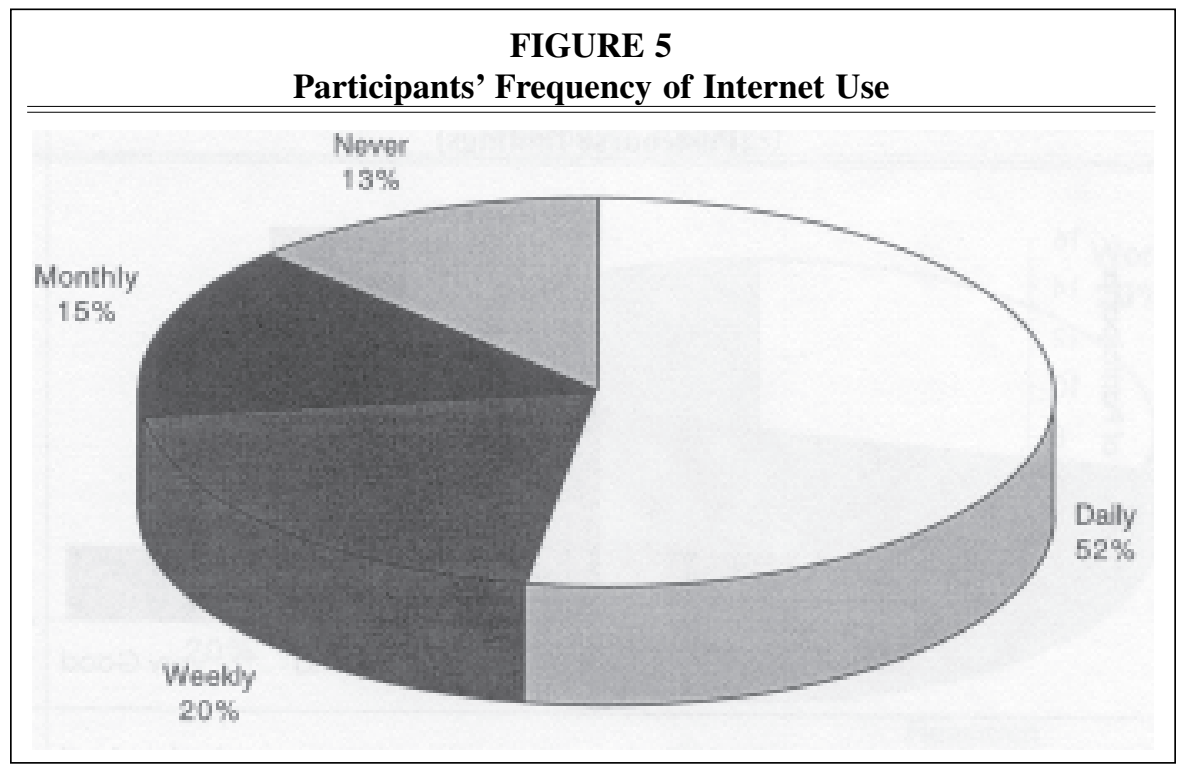

marily for research and work (see figure 8). Compared to the precourse survey, the postcourse survey showed an increase in Internet use for personal use (see figure 9). The increase in using the
Internet for personal use shows the benefit achieved in taking the course as it increased the comfort and familiarity level of the participants in their plans for Internet use.

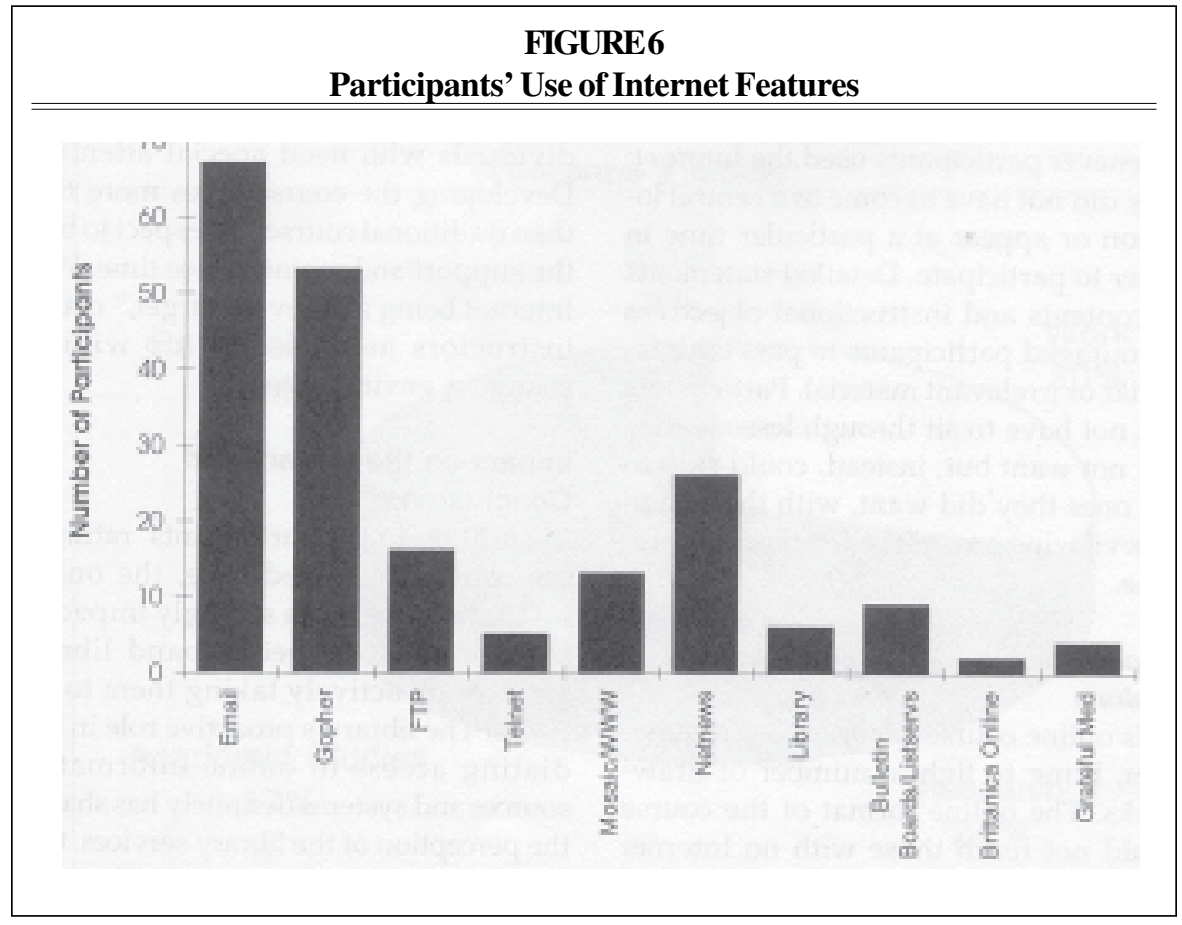




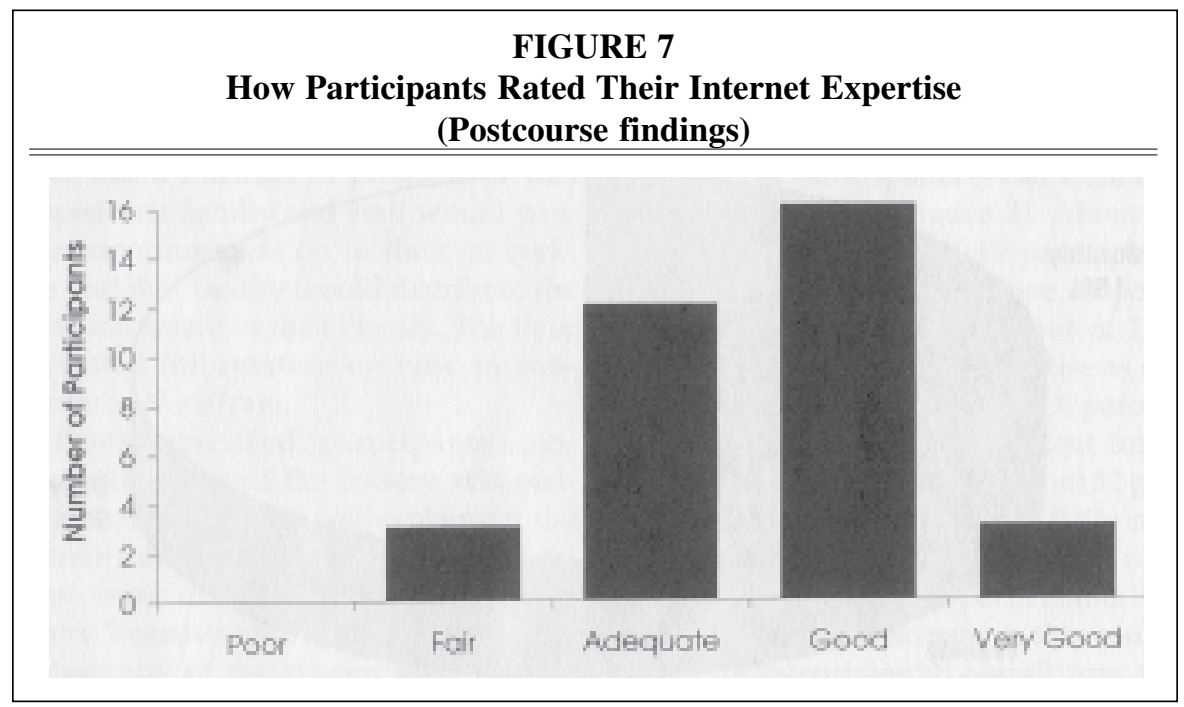

The surveys showed that participants built on their familiarity with email to broaden their Internet expertise. As the course was devised to make instruction more accessible to users when they wanted help, participants preferred the e-mail workshop format of the course $(83 \%)$ over the traditional classroom setting because of the convenience of attending the session at their desktops and having the flexibility of reading the lessons at any time.

\section{Advantages of the Online Instruction Medium}

UIC is a campus where most students are commuters, and a large percentage of students, staff, and faculty already have access to the campus electronic mail network and Internet. Thus, the fact that distributing lessons by email permitted participants to go through them at their convenience was definitely advantageous. Online instruction limited participation to those who already had Internet access but did not eliminate those who would have been unable to meet at a particular time or place. It thus permitted instruction to take place wherever and whenever participants used the Internet; they did not have to come to a central location or appear at a particular time in order to participate. Detailed statements of contents and instructional objectives encouraged participants to pass over familiar or irrelevant material. Participants did not have to sit through lessons they did not want but, instead, could skip to the ones they did want, with the option of reviewing any of the lessons at a later time.

\section{Disadvantages of the Online Instruction Medium}

This online course experience did, however, bring to light a number of drawbacks. The online format of the course could not reach those with no Internet experience or without Internet access. Online instruction reached only those users who already had Internet access, except in few cases. Though feedback was available via a listserv open to all participants, there was a lack of physical contact, which sometimes can be more effective. Overcoming anxiety and unfamiliarity with computers in general might require a hands-on approach, and in such an online instruction environment it is not possible to reach those individuals who need spe- 


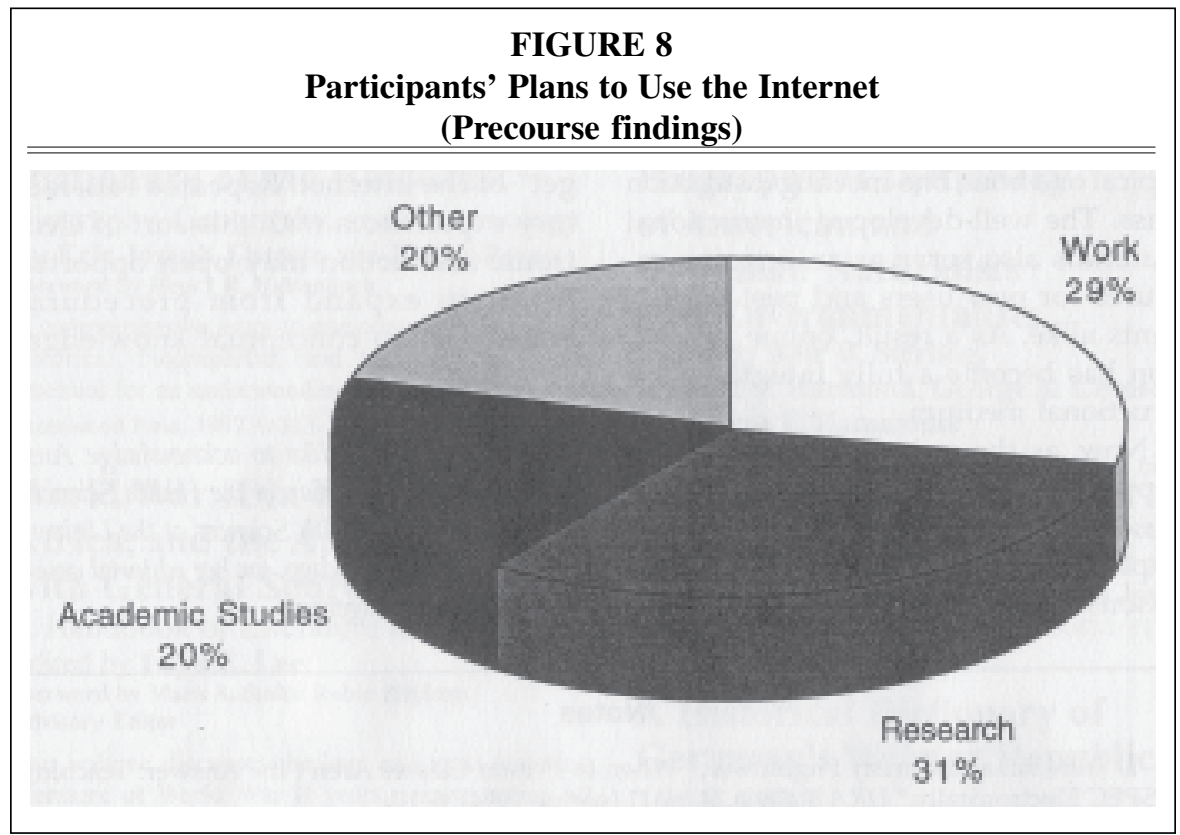

cial attention. Developing the course takes more time than traditional courses in respect to both the support and maintenance time. With Internet being a "moving target," course instructors need to keep up with its changing environment.

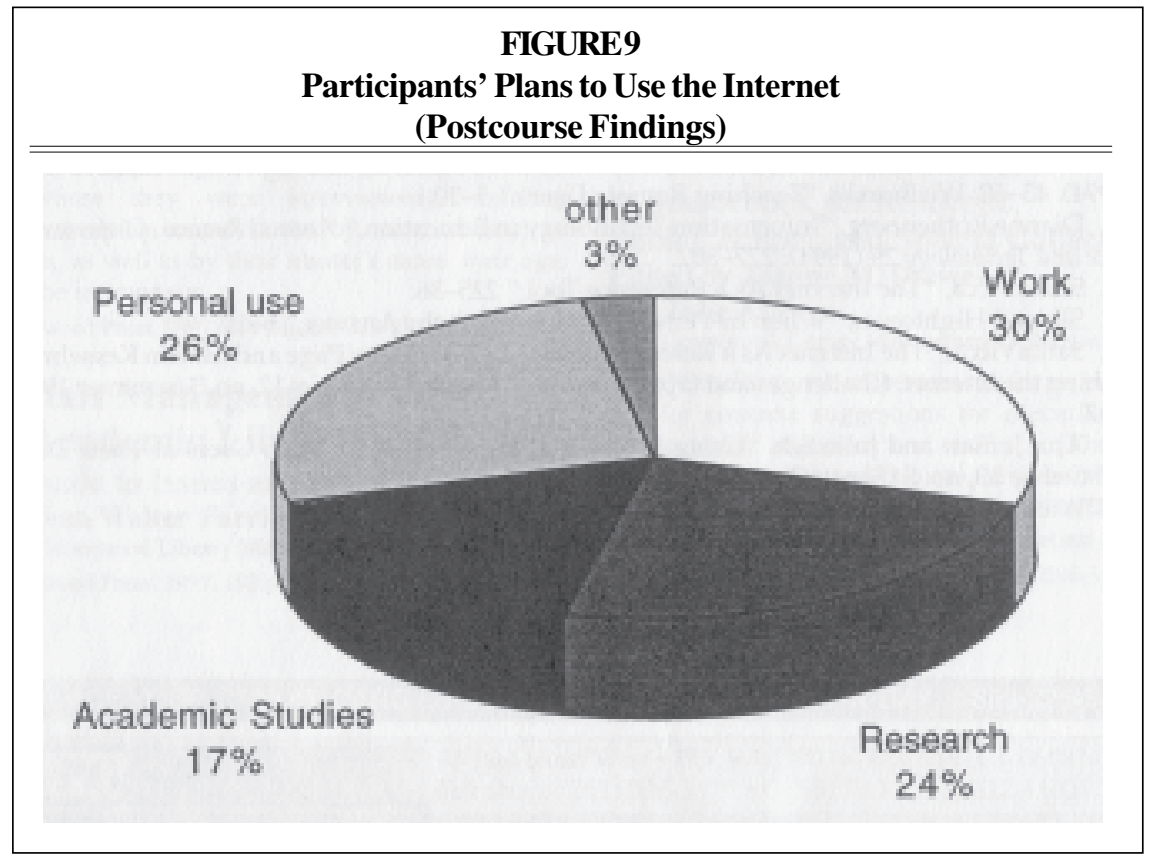




\section{Impact on the Library and Conclusions}

In addition to the participants' rating of the course described here, the online instruction medium strongly impacted the library. It helped expand library services by actively taking them to the users. The library's proactive role in mediating access to online information sources and systems definitely has shaped the perception of the library services. One of the most important impacts of this course is the pointof-use support in a remote setting.

Although a number of disadvantages were noted, the overall experience of offering online instruction was positive for both users and library staff. A large number of users benefited from the program. The online medium allowed the library to reach a larger number of users than would have been possible in the classroom setting, while also providing a level of detail and depth not possible in the typical one-hour, onemeeting instruction class. The well-de- veloped instructional materials also serve as an ongoing resource for new users and past participants alike. As a result, online instruction has become a fully integrated instructional medium.

Now, as the university increasingly supports nonmainframe Internet access, various access platforms will be included in plans for revisions and supplemental lessons. Once structures are in place for delivering instruction online, it may be possible to meet the needs of the "moving target" of the remote user for information on using the "moving target" of the Internet. Repeated satisfactory experiences with this sort of electronic instruction may open opportunities to expand from procedural knowledge to conceptual knowledge. Time will tell.

The authors would like to acknowledge Ann Weller, Deputy Librarian of the Health Sciences in the Library of Health Sciences at the University of Illinois at Chicago, for her editorial assistance and encouragement.

\section{Notes}

1. Julie Sih and Christy Hightower, "When In-Person Classes Aren't the Answer: Teaching INSPEC Electronically," DRA Bulletin 14, no. 1 (spring 1994): 6-10.

2. Karen Wielhorski, "Teaching Remote Users How to Use Electronic Information Resources," Public-Access Computer Systems Review 5, no. 4 (1994): 5-20.

3. Sih and Hightower, "When In-Person Classes Aren't the Answer," 6-10; Wielhorski, "Teaching Remote Users," 5-20. $54-55$.

4. John S. Makulowich, "Meeting the Demands of Internet Training," Online 19, no. 4 (1995):

5. Lisa A. Oberg, "The Moving Target: Internet Instruction at the University of Washington Health Sciences Libraries and Information Center," Medical Reference Services Quarterly 15, no. 3 (fall 1996): 73-79.

6. Wielhorski, “Teaching Remote Users," 5-20.

7. Edmund F. SantaVicca, "The Internet As a Reference Tool: A Model for Educators," Reference Librarian 41/42 (1994): 225-36.

8. Wielhorski, "Teaching Remote Users," 5-20.

9. Susan J. Barnes, "The Electronic Library and Public Services," Library Hi Tech 12, no. 3 (winter 1994): 43-62; Wielhorski, "Teaching Remote Users," 5-20.

10. Dianne Rothenberg, "Information Technology in Education," Annual Review of Information Science and Technology 29 (1994): 277-302.

11. SantaVicca, "The Internet As a Reference Tool," 225-36.

12. Sih and Hightower, "When In-Person Classes Aren't the Answer," 6-10.

13. SantaVicca, "The Internet As a Reference Tool," 225-36; Mary Page and Martin Kesselman, "Teaching the Internet: Challenges and Opportunities," Research Strategies 12, no. 3 (summer 1994): 157-67.

14. Ann Jensen and Julie Sih, "Using E-mail and the Internet to Teach Users at Their Desktops," Online 19, no. 5 (Sept./Oct. 1995): 83-86.

15. Wielhorski, “Teaching Remote Users," 5-20. 